\title{
Simplified bond-hyperpolarizability model of second harmonic generation: Application to Si-dielectric interfaces
}

\author{
J.-F. T. Wang, G. D. Powell, R. S. Johnson, G. Lucovsky, and D. E. Aspnes ${ }^{\text {a) }}$ \\ Department of Physics, North Carolina State University, Raleigh, North Carolina 27695-8202
}

(Received 12 February 2002; accepted 22 April 2002)

\begin{abstract}
We show that the anisotropies of second-harmonic-generation (SHG) intensities of singular and vicinal (111) and (001)Si-dielectric interfaces can be described accurately as dipole radiation originating from the anharmonic motion of bond charges parallel to the bond directions. This simplified bond-hyperpolarizability model not only provides a simpler and mathematically more efficient representation of SHG, but also allows a direct physical interpretation at the bond level, which was lacking in previous approaches. Application to oxidized and nitrided $\mathrm{Si}-\mathrm{SiO}_{2}$ interfaces provides new insight into bonding that occurs at these interfaces as well as the origin of SHG.

(C) 2002 American Vacuum Society. [DOI: 10.1116/1.1493783]
\end{abstract}

\section{INTRODUCTION}

The development of the femtosecond laser has generated a significant resurgence of interest in the use of nonlinearoptical (NLO) phenomena as a means of studying bulk materials, thin films, interfaces, and surfaces. ${ }^{1,2}$ NLO phenomena possess richer selection rules than their linear-optic equivalent, and are therefore intrinsically more powerful as diagnostic tools. ${ }^{1-3}$ In addition, the microscopic symmetry of surfaces and interfaces is lower than that of bulk material, which can provide an additional means of isolating contributions from these regions. For example, with the exception of the bulk quadrupole contribution, which is relatively small in materials such as $\mathrm{Si}$ that possess inversion symmetry, second-harmonic generation (SHG) is forbidden in the bulk and also in amorphous materials such as $\mathrm{SiO}_{2}$ overlayers where the bonds are oriented in essentially random directions. Thus SHG of oxidized or nitrided Si wafers originates almost entirely from an interface region no more than several atomic layers thick, where there is a regular repeating geometry of asymmetric bonds. As a result SHG has become a widely used probe for studying the interfaces of this technologically important materials system., ${ }^{1,2,4-12}$

However, NLO spectra are difficult to acquire, so with few exceptions ${ }^{12}$ SHG data have been limited to relatively narrow spectral ranges and are typically obtained as anisotropies $I_{\mathrm{SHG}}(\phi)$ of the second-harmonic intensity $I_{\mathrm{SHG}}$ as a function of the sample azimuth angle $\phi$. Since the associated nonlinear susceptibility $\chi_{\mathrm{SHG}}=\chi_{2}$ is a third-rank tensor, anisotropies up to and including $3 \phi$ may be analyzed by this approach. To organize these data the results are typically expressed either as the coefficients of the Fourier expansion of $I_{\mathrm{SHG}}(\phi)$ or as tensor coefficients, taking account of grouptheoretical constraints ${ }^{13-15}$ to eliminate all those that are not allowed by crystal symmetry. Neither approach is satisfactory, since there is no obvious connection between the Fourier or tensor coefficients and the properties of the individual bonds. Moreover, the absolute-squaring operation that con-

a)Author to whom correspondence should be addressed; electronic mail: aspnes@unity.ncsu.edu verts fields to intensities generates additional Fourier coefficients and obscures phases, further complicating analysis. Interpretation on the basis of available first-principles expressions involves lengthy calculations ${ }^{16}$ and a significant loss of physical insight. This situation has created a barrier to the more widespread use of SHG.

To gain the information about the interface chemistry and bonding on a microscopic scale that we needed, a better approach was necessary. Because the region from which SHG originates in the Si-dielectric system is localized, it seemed that a much more sensible method of analysis was to formulate SHG in terms of its basic physics, starting at the bond rather than the band level and representing observed SHG intensities as far-field radiation from dipoles driven anharmonically by the incident electric field. This approach employs the nonlinear-optical equivalent of techniques that were used by Ewald ${ }^{17}$ and Oseen ${ }^{18}$ in their derivations of the extinction theorem of linear optics. Using physically reasonable approximations we found that we could indeed develop a simple approach that not only replaces the cataloging of phenomenological quantities but also allows SHG data to be represented by a minimum number of parameters, each of which has a direct physical interpretation at the bond level. ${ }^{19}$ For example, we show below that we not only can recognize SHG absorption from the anisotropy of $I_{\mathrm{SHG}}(\phi)$ but also identify the responsible bonds. Because these parameters can also be calculated from first principles, our framework also provides a convenient interface between experiment and theory. We anticipate that our approach will stimulate a resurgence of interest in SHG.

Specifically, we assume that the system consists of charges localized in bonds, then follow a three-step procedure. First, we recognize that the applied field causes a displacement of charge (dipole) at each bond site, which varies anharmonically with time under the action of the applied field and appropriate restoring and dissipation forces. We summarize the linear and nonlinear parts of this motion in terms of complex polarizabilities and hyperpolarizabilities, respectively, which can be obtained in principle by solving the standard equation of motion as discussed by Shen. ${ }^{3}$ Next, 
we make the simplifying assumption that for any bond the only relevant anharmonic motion is that along the bond axis. In the SHG context this is equivalent to assuming that the bonds are cylindrically symmetric. Finally, we calculate the far-field intensity as the square of the superposition of fields radiated by these charges in the dipole approximation, assuming that the relevant bond directions are those of the underlying bulk crystalline material. This simplified bondhyperpolarizability model (SBHM) leads to an excellent description of SHG signals from (111) and (001) Si-dielectric interfaces, and in addition provides new physical insight at the microscopic level not only about these interfaces but also about the physics of SHG itself.

The new aspect with respect to Ewald ${ }^{17}$ and Oseen ${ }^{18}$ beyond the application to a nonlinear process, and with respect to early NLO bond calculations of the dc susceptibility, ${ }^{20-22}$ is our assumption that for SHG purposes we need to consider bond polarization only along bond directions. This also distinguishes the present approach from more recent bondorbital treatments of SHG in covalent materials, ${ }^{23,24}$ including that of Wijers et al. where SHG was also formulated in terms of radiating bonds. Cluster calculations for Asterminated $\mathrm{Si}$ and Ge surfaces showed that the assumption of cylindrical symmetry for individual bonds is not necessarily a good approximation, ${ }^{25}$ but we justify its use because we find that our model accurately represents SHG data with an absolute minimum number of parameters, thereby implying either simplifications or outright cancellations in what is basically an effective-bond representation. A bondhyperpolarizability model similar to ours, including the assumption of cylindrical symmetry for the individual bonds, was recently presented by Mendoza and co-workers. ${ }^{26,27}$ However, Mendoza et al. represented SHG as a product of linear polarizabilities for which the transverse polarizability cannot be ignored, and hence overlooked the essential simplification that results from the assumption of cylindrical symmetry. Since this assumption cannot be made for linear optics, we conclude that in at least some aspects SHG is simpler than linear optics.

\section{THEORY}

\section{A. Formulation}

We outline only basic aspects since a more complete description has been published elsewhere. ${ }^{19}$ Consistent with our assumption that only motion along the bond axis need be considered, we examine a one-dimensional force model as discussed by Shen. ${ }^{3}$ Let the direction of the $j$ th bond in a unit cell or other appropriate set of bonds be defined by the unit vector $\hat{\mathbf{b}}_{j}$. We suppose an applied field $\mathbf{E} e^{-i \omega t}$, noting that the field $\mathbf{E}_{j}$ at the $j$ th bond site may be different from $\mathbf{E}$ owing to contributions from other induced dipoles in the area (local-field effect). However, for simplicity we assume that $\mathbf{E}_{j}=\mathbf{E}$. Then the motion of the charge $q_{j}$ can be described by the equation of motion:

$$
\begin{aligned}
F & =q_{j} \mathbf{E} \cdot \hat{\mathbf{b}}_{j} e^{-i \omega t}-\kappa_{1}\left(x-x_{o}\right)-\kappa_{2}\left(x-x_{o}\right)^{2}-\gamma d x / d t \\
& =m d^{2} x / d t^{2},
\end{aligned}
$$

where $x$ is the position of the charge in a local coordinate system that lies along the $\hat{\mathbf{b}}_{j}$ direction, $x_{o}$ is the equilibrium position, $\kappa_{1}$ and $\kappa_{2}$ are the harmonic (Hooke's law) and anharmonic spring constants, respectively, and $\gamma$ is a frictional coefficient representing losses. Assuming that $x$ can be written

$$
x=x_{o}+\Delta x_{1} e^{-i \omega t}+\Delta x_{2} e^{-i 2 \omega t},
$$

it follows that to lowest order the linear $\mathbf{p}_{1 j}$ and first-order nonlinear $\mathbf{p}_{2 j}$ parts of the induced dipole $\mathbf{p}_{j}=q_{j} \Delta \mathbf{x}_{j}$ corresponding to the $j$ th charge are given in a general coordinate system by

$$
\begin{aligned}
& \mathbf{p}_{1 j}=q_{j} \Delta x_{1} \hat{\mathbf{b}}_{j}=\frac{q_{j}^{2} \mathbf{E} \cdot \hat{\mathbf{b}}_{j}}{\kappa_{1}-m \omega^{2}-i \gamma \omega} \hat{\mathbf{b}}_{j}=\alpha_{1 j} \hat{\mathbf{b}}_{j}\left(\hat{\mathbf{b}}_{j} \cdot \mathbf{E}\right), \\
& \mathbf{p}_{2 j}=q_{j} \Delta x_{2} \hat{\mathbf{b}}_{j}=\frac{q_{j} \kappa_{2} \Delta x_{1}^{2}}{\kappa_{1}-4 m \omega^{2}-2 i \gamma \omega} \hat{\mathbf{b}}_{j}=\alpha_{2 j} \hat{\mathbf{b}}_{j}\left(\hat{\mathbf{b}}_{j} \cdot \mathbf{E}\right)^{2},
\end{aligned}
$$

where $\alpha_{1 j}$ and $\alpha_{2 j}$ are the microscopic first-order (linear) polarizability and second-order (first-order nonlinear) hyperpolarizability, respectively, of the $j$ th bond.

Thus, to within a scaling factor, the total polarization can be written

$$
\begin{aligned}
\mathbf{P}=\frac{1}{V} \sum_{j} \mathbf{p}_{j}= & \frac{1}{V} \sum_{j}\left(\alpha_{1 j} \hat{\mathbf{b}}_{j} \hat{\mathbf{b}}_{j}\right) \cdot \mathbf{E} \\
& +\frac{1}{V} \sum_{j}\left(\alpha_{2 j} \hat{\mathbf{b}}_{j} \hat{\mathbf{b}}_{j} \hat{\mathbf{b}}_{j}\right) \cdot \mathbf{E E} \\
= & \chi_{1} \cdot \mathbf{E}+\chi_{2} \cdot \mathbf{E E},
\end{aligned}
$$

where $V$ is the volume and $\chi_{1}$ and $\chi_{2}$ are the first- and second-order susceptibility tensors, respectively, of the system. Using the assumption that the bond charges radiate as dipoles, we can now express the far-field radiation field $\mathbf{E}_{f f}$ as

$$
\begin{aligned}
\mathbf{E}_{f f} & =k^{2} \frac{e^{i k r}}{r}\left(\sum_{j} \mathbf{p}_{j}-\hat{\mathbf{k}}\left[\hat{\mathbf{k}} \cdot \sum_{j} \mathbf{p}_{j}\right]\right) \\
& =k^{2} \frac{e^{i k r}}{r}(\mathbf{I}-\hat{\mathbf{k}} \hat{\mathbf{k}}) \cdot \sum_{j} \mathbf{p}_{j},
\end{aligned}
$$

where $\mathbf{I}$ is the unit tensor and $\mathbf{k}=k \hat{\mathbf{k}}$ is the wave vector pointing in the direction of the observer. By combining Eqs. (4) and (5b) it is clear that in this model the different orders of susceptibilities can be defined in intrinsic terms as sums of dyadic, triadic, etc. products of the bonds, with the description of the observed radiation and the action of the applied fields both represented as external operators. Extensions to higher orders are obvious.

We consider here analytic expressions for SHG signals only from singular (111) interfaces; (001) interfaces can be treated similarly. Here, we have one "up" bond and three 
equivalent "back" bonds, although for vicinal surfaces one of the three back bonds becomes an inequivalent step bond. Choosing a coordinate system where one axis is perpendicular to the interface and another parallel to the step, these bonds can be written: up: $\hat{\mathbf{b}}_{1}=\hat{\mathbf{z}} ; \quad$ step: $\hat{\mathbf{b}}_{2}=(\sqrt{8}) / 3 \hat{\mathbf{x}}$ $-(1 / 3) \hat{\mathbf{z}}$; back: $\hat{\mathbf{b}}_{3,4}=-(\sqrt{2}) / 3 \hat{\mathbf{x}} \pm(\sqrt{6}) / 3 \hat{\mathbf{y}}-(1 / 3) \hat{\mathbf{z}}$.

We suppose that all bonds point away from the $\mathrm{Si}$ atom in the outermost plane. If the incoming angle of incidence is $\theta_{i}$, the incoming $s$ - and $p$-polarized beams are $\mathbf{E}_{s}=E_{s} \hat{\mathbf{y}}$ and $\mathbf{E}_{p}=E_{p}\left(-\hat{\mathbf{x}} \cos \theta_{i}+\hat{z} \sin \theta_{i}\right)$, respectively. If the observation angle is $\theta_{o}$, the $\mathbf{k}$ vector of the outgoing wave is $\hat{\mathbf{k}}$ $=-\hat{\mathbf{x}} \sin \theta_{o}+\hat{\mathbf{z}} \cos \theta_{o}$. In this case the far fields $\mathbf{E}_{f f}$ observed in the four possible combinations of polarization are given for the $p-p$ case:

$$
\begin{aligned}
\mathbf{E}_{f f}= & {\left[\hat{\mathbf{x}} \cos \theta_{o}+\hat{\mathbf{z}} \sin \theta_{o}\right]\left[\alpha_{u} \sin ^{2} \theta_{i} \sin \theta_{o}\right.} \\
& +\alpha_{d}\left(\cos ^{3} \beta \sin ^{2} \theta_{i} \sin \theta_{o}\right. \\
& +\frac{3}{4} \sin \beta \sin 2 \beta\left(\cos ^{2} \theta_{i} \sin \theta_{o}-\sin 2 \theta_{i} \cos \theta_{o}\right) \\
& \left.\left.+\frac{3}{4} \sin ^{3} \beta \cos ^{2} \theta_{i} \cos \theta_{o} \cos 3 \phi\right)\right],
\end{aligned}
$$

for the $p-s$ case:

$$
\mathbf{E}_{f f}=\hat{\mathbf{y}} \frac{3}{4} \alpha_{d} \sin ^{3} \beta \cos ^{2} \theta_{i} \sin 3 \phi,
$$

for the $s-p$ case:

$$
\begin{aligned}
\mathbf{E}_{f f}= & {\left[\hat{\mathbf{x}} \cos \theta_{o}+\hat{\mathbf{z}} \sin \theta_{o}\right]\left(\frac { 3 } { 4 } \alpha _ { d } \left(-\sin ^{3} \beta \cos \theta_{o} \cos 3 \phi\right.\right.} \\
& \left.\left.+\sin \beta \sin 2 \beta \sin \theta_{o}\right)\right),
\end{aligned}
$$

for the $s-s$ case:

$$
\mathbf{E}_{f f}=-\hat{\mathbf{y}} \frac{3}{4} \alpha_{d} \sin ^{3} \beta \sin 3 \phi,
$$

where $\beta=109.47^{\circ}$ is the angle between bonds and $\phi$ is the azimuth angle measured from the $x z$ plane. To calculate the intensity we take the absolute square of these fields.

For (001) interfaces the situation is complicated by the fact that real on-axis interfaces are not singular but consist of statistically equal areas of two inequivalent domains that are rotated by $90^{\circ}$ with respect to each other. Thus except for the dc component the terrace contributions vanish, leaving that from the steps. In a full least-squares analysis for either orientation we do not rely on analytic expressions but rather evaluate Eqs. (4a) and (5b) numerically, with all bonds tilted appropriately in the case of vicinal surfaces.

\section{B. Validation: Analysis of oxidized vicinal (111) and (001) Si}

We assess the validity of the model by applying it to the data of Lüpke, Bottomley, and van Driel on an oxidized vicinal (111) Si wafer reported in Ref. 8. These data are appropriate because all four combinations of polarizations are given. However, the amplitudes of the measured SHG anisotropies are normalized to the $3 \phi$ Fourier component, which means that the information that in principle is available from the relative amplitudes of the four configurations is lost, and that comparisons must be done on the basis of line

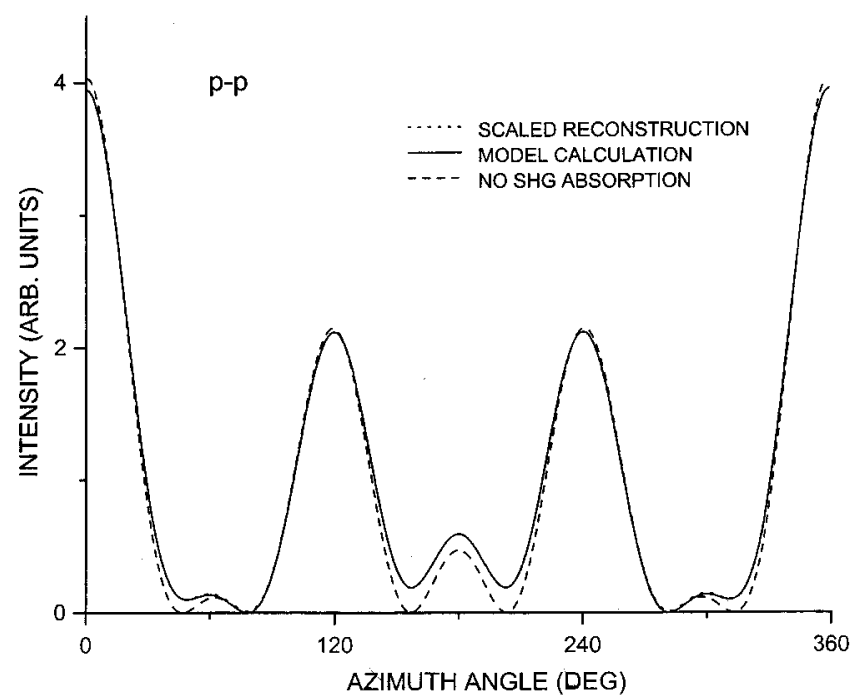

FIG. 1. Comparison between the reconstructed $p$ - $p$ SHG data of Lüpke et al. for a $5^{\circ}$ vicinal (111) $\mathrm{Si}-\mathrm{SiO}_{2}$ interface (points, Ref. 8) and the variation obtained with the SBHM using the best-fit parameters given (solid line). These are essentially indistinguishable on the scale of the figure. Also shown is the best-fit result obtained if SHG absorption is assumed to be zero.

shapes alone. The wavelength $\lambda$ was $765 \mathrm{~nm}$, the nominal miscut angle $5^{\circ}$ toward the $[11 \overline{2}]$ direction, and the nominal angles of incidence and observation $45^{\circ}$. Setting aside the normalization coefficients, the data are presented as a table of seven real and two complex Fourier coefficients (Table VI of Ref. 8), which are also given apart from normalization as seven complex coefficients of $\chi_{2}$ (Table VII). Using their Fourier coefficients we have reconstructed their data as intensities, normalizing them in our case to the $6 \phi$ coefficients.

We determine the (complex) bond hyperpolarizabilities by least-squares fitting the azimuthal variation of the SHG intensity for the $p$ - $p$ configuration. The result, shown in Fig. 1, is an essentially exact representation of the reconstructed data. The least-squares values obtained are $29.5^{\circ}$ for the angles of incidence and observation, $1.12^{\circ}$ for the vicinal angle, and $1.78+i 0.17,2.76+i 1.43,2.19+i 0.00$, and 2.19 for the hyperpolarizabilities of the up, step, and two back bonds, respectively. The imaginary part of the hyperpolarizability of the last back bond is arbitrarily set equal to zero, since the absolute phase cannot be determined in an intensity measurement. The values for the back bonds are equivalent, as expected. We find therefore that, again apart from normalization, we can essentially represent the $p$ - $p$ line shape with three parameters, one less than the number of Fourier coefficients needed to describe the same data in Ref. 8. Thus the SBHM not only has the mathematical breadth necessary to represent SHG anisotropies, but also does it in a more efficient and physically more meaningful manner.

The existence of SHG absorption can be demonstrated unequivocally by repeating the least-squares fit with the imaginary parts of the hyperpolarizabilities of the first two bonds set equal to zero. The result is the dashed line in Fig. 1 , which is obtained with the least-squares values 1.743, $3.158,2.230$, and 2.230. More to the point, in the absence of 

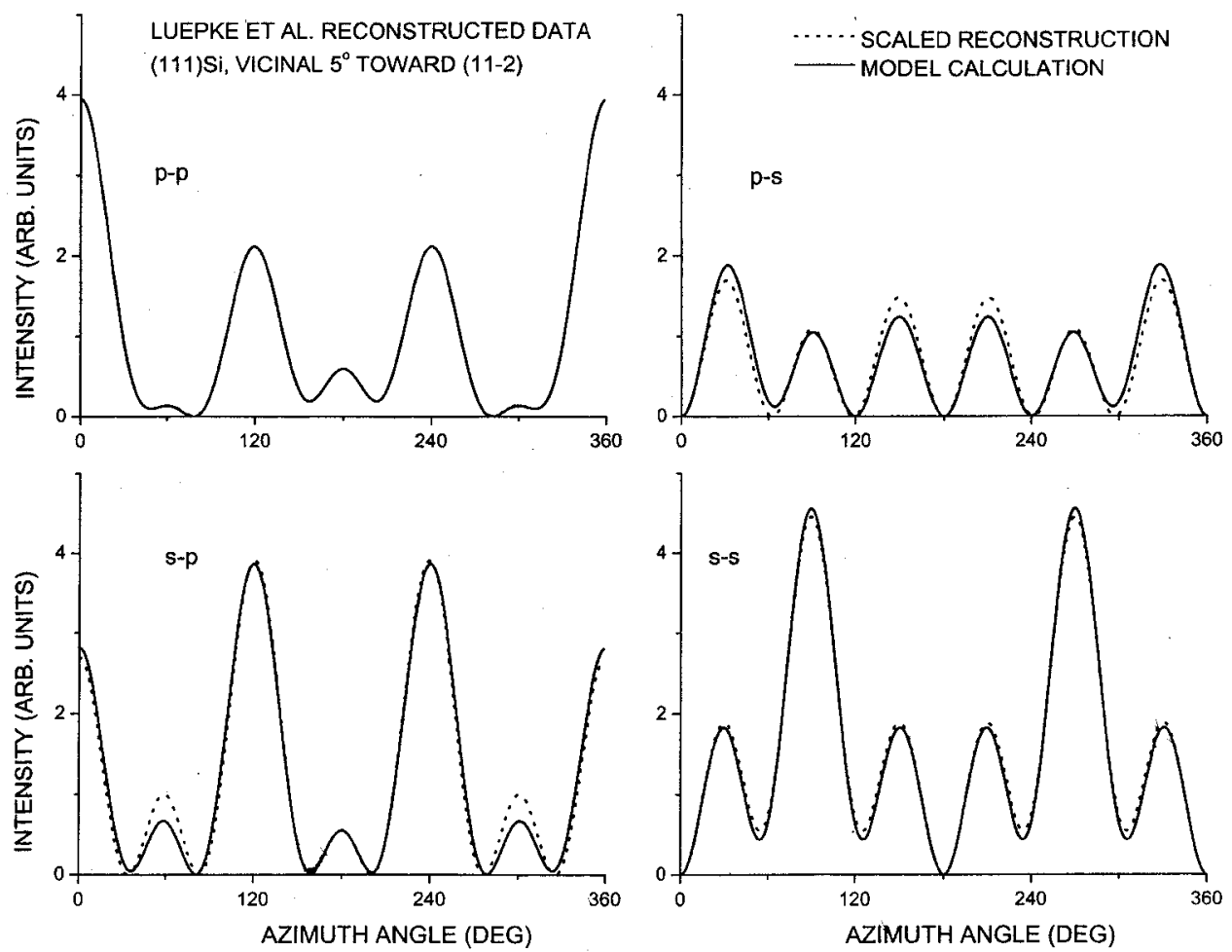

FIG. 2. Comparisons among all reconstructed SHG data of Lüpke et al. (points, Ref. 8) using the parameters determined for the $p$ - $p$ configuration in Fig. 1, which is reproduced in the upper left quadrant. Scaling factors of 1.40, 1.93, and 2.26 were used for the $p-s, s-p$, and $s$-s dependencies.

SHG absorption the SHG intensity reaches zero at the local minima near $80^{\circ}, 155^{\circ}, 205^{\circ}$, and $280^{\circ}$. Thus, SHG absorption is relatively easy to recognize from these data.

Taking the best-fit $p-p$ parameters we now calculate the expected intensity anisotropies for the $p-s, s-p$, and $s-s$ configurations. The reconstructed data and least-squares fits are shown in Fig. 2. As can be seen, the SBHM provides a very good representation of these data, provided that the predicted line shapes are scaled by the least-squares-determined values $1.40,1.93$, and 2.26, respectively. The need for, and physical meaning of, these scaling factors can be understood by noting that the data shown in Fig. 2 were scaled to the $6 \phi$ Fourier component. From Eqs. (6) we see that if the angle of incidence $\theta_{i}$ equals the angle of observation $\theta_{o}$, the $6 \phi$ anisotropies of the four configurations differ only in that the $p-s, s-p$, and $s-s$ coefficients are multiplied by $1 / \cos ^{2} \theta$, $1 / \cos ^{4}(\theta)$, and $1 / \cos ^{6}(\theta)$, respectively, where $\theta=\theta_{i}=\theta_{o}$. From the scaling factors determined above we find $\theta$ values of $32.5^{\circ}, 32.0^{\circ}$, and $29.2^{\circ}$ for the $p-s, s-p$, and $s-s$ configurations, respectively, essentially equal to expected values. We also note that the least-squares-determined value of $29.5^{\circ}$ for $\theta=\theta_{i}=\theta_{o}$ is in excellent agreement with the values of $29.1^{\circ}$ and $28.7^{\circ}$ calculated for refraction at the air $-\mathrm{SiO}_{2}$ interface using the refractive indices of 1.454 and 1.472 for $\mathrm{SiO}_{2}$ at 765 and $382.5 \mathrm{~nm}$, respectively. From this we can also conclude that for this oxidized (111)Si interface the SHG signal originates essentially from the $\mathrm{SiO}_{2}$ side of the interface. Since we can account for the scaling parameters, the SBHM reconstructs the $p-s, s-p$, and $s-s$ anisotropies with the same three parameters determined for the $p-p$ configuration, in contrast to the seven additional Fourier coefficients and 14 overall tensor coefficients needed in Ref. 8.

For the oxidized vicinal (001) Si interface similar good agreement is obtained, as shown by our data in Fig. 3. This sample was prepared by thermally growing a $10 \mathrm{~nm}$ sacrificial oxide at $900^{\circ} \mathrm{C}$, stripping the oxide with $\mathrm{HF}$, then thermally regrowing a $3 \mathrm{~nm}$ oxide at $750^{\circ} \mathrm{C}$ by annealing at $900^{\circ} \mathrm{C}$. The angles of incidence and observation were $45^{\circ}$ and the wavelength was $830 \mathrm{~nm}$. The $p$-s data were multiplied by a factor of 4 to compensate for reflectance effects and different pulse durations and intensities that resulted as a consequence from the need to use different experimental conditions with respect to the $p$-incident configuration. The least-squares fitting parameters were determined to be $12^{\circ}$ for the angles of incidence and observation, $7.28^{\circ}$ for the vicinal angle, and $105+i 30,197+i 55,133+i 0$, and 131 for the hyperpolarizabilities of the four bonds, again in arbitrary units and with the phase of the hyperpolarizability of the last bond arbitrarily set equal to zero. The "step" bond again shows the highest polarizability and absorption, although the essentially threefold pattern of Fig. 2 shows that most of the signal is coming from the vicinity of the steps. The $p-s$ anisotropy is found to be particularly sensitive to the angles of incidence and observation, and we estimate that the $12^{\circ}$ value is determined to within $3^{\circ}$. This angle is consistent with Snell's Law for a refractive index of 3.4, which indicates that the SHG signal here is originating predominantly from the Si side of the interface. 


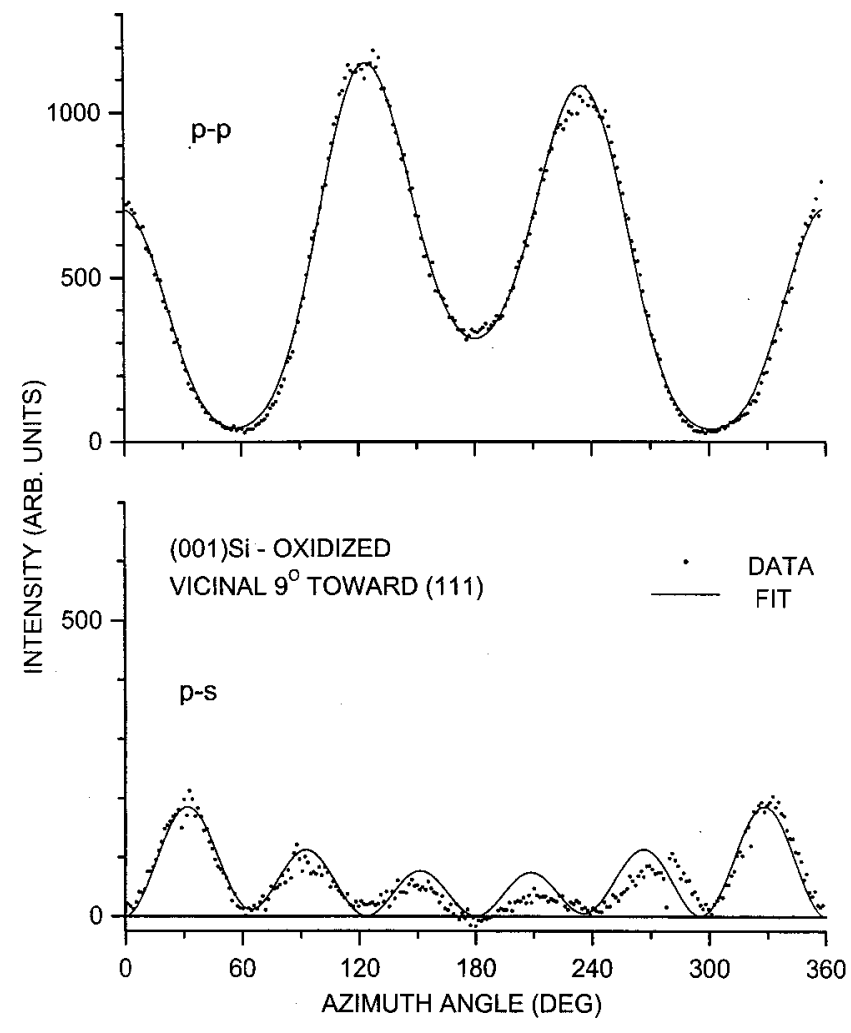

FIG. 3. Result of least-squares fitting for a $9^{\circ}$ vicinal $\mathrm{Si}-\mathrm{SiO}_{2}$ interface.

\section{APPLICATION TO PLASMA-DEPOSITED AND RAPID-THERMAL-ANNEALED INTERFACES}

We have performed a number of measurements on interfaces formed on (111) Si surfaces by plasma oxidation and nitridation followed by rapid thermal annealing (RTA). The data shown in Fig. 4 are for an oxidized surface cut $5^{\circ}$ off (111) toward $(11 \overline{2})$. The incident wavelength was $830 \mathrm{~nm}$, and measurements were made in situ, that is, without exposing the sample to air. Data for $(\overline{112})$ and nitrided samples are similar. The anisotropy follows the typical pattern shown in Fig. 1, with a basic threefold waveform superimposed on a dc background, which when squared gives rise in a Fourier expansion primarily to dc, $3 \phi$, and $6 \phi$ components. Qualitatively RTA processing reduces SHG absorption and increases the magnitude of the hyperpolarizability of the up bond relative to the other three.

The complex hyperpolarizabilities obtained from a leastsquares fit of the SBHM to these data, and those obtained for related surfaces, are summarized in Table I. We show these data in polar (amplitude, angle) rather than rectangular (real, imaginary) form because the polar format better illustrates the fact that the amplitudes of the hyperpolarizabilities appear to be generally unaffected by RTA while the absorption decreases. The phases are defined according to our previous convention, i.e., that the phase of one of the back bonds is arbitrarily set equal to zero. The fact that some of the stepbond phases are now slightly negative indicates that this assumption is not strictly valid in all cases. The hyperpolarizabilities of the oxidized (112) sample are seen to support the

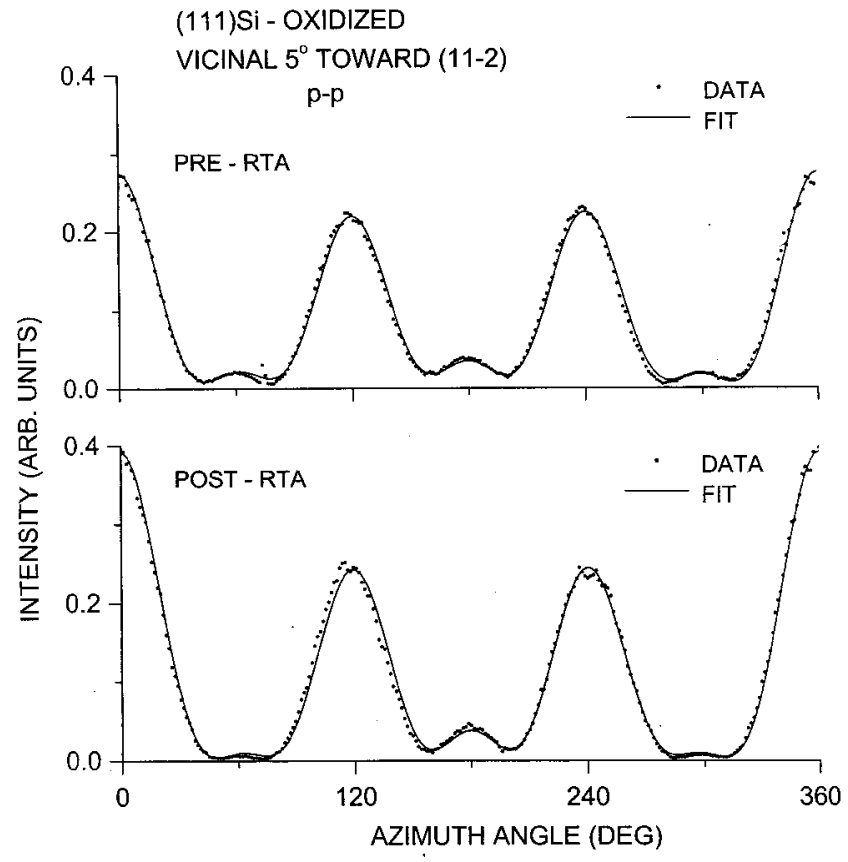

FIG. 4. Comparison of SHG anisotropies before and after an oxidized (11̄ $)$ sample has undergone rapid thermal annealing. The corresponding hyperpolarizabilities for this and other Si-dielectric interfaces are listed in Table I.

qualitative interpretation given above, in that the SHG absorption, here associated primarily with the up bond, is decreased significantly by RTA. This is consistent with the conversion of the interface suboxide to $\mathrm{Si}$ and $\mathrm{SiO}_{2}$, a known consequence of rapid thermal annealing. The hyperpolarizability of the step bond has also increased, further indicating improved bonding. The hyperpolarizabilities of the two back bonds, which as expected are essentially identical, are basically unchanged by RTA.

The response of the nitrided $(11 \overline{2})$ interface is very similar. The dominant hyperpolarizability is again seen to occur for the up bond, and the major fraction of SHG absorption occurs for this bond as well. As with the oxidized interface, RTA reduces the SHG absorption for the up bonds and increases the hyperpolarizability for the step bonds. Both indicate improved bonding as a result of RTA.

TABLE I. Hyperpolarizabilities obtained for plasma-processed vicinal (111) Si samples before and after rapid thermal annealing.

\begin{tabular}{lclc}
\hline \hline Orientation and procedure & $\mathrm{Up}$ & \multicolumn{1}{c}{ Step } & Back \\
\hline$(11 \overline{2})$ oxidation, pre-RTA & $1.09 / 55^{\circ}$ & $0.79 /-4^{\circ}$ & 0.66 \\
$(11 \overline{2})$ oxidation, post-RTA & $1.12 / 38^{\circ}$ & $0.92 /-6^{\circ}$ & 0.67 \\
$(11 \overline{2})$ nitridation, pre-RTA & $1.16 / 49^{\circ}$ & $0.75 /-6^{\circ}$ & 0.72 \\
$(\overline{11 \overline{2}})$ nitridation, post-RTA & $1.17 / 35^{\circ}$ & $0.85 /-6^{\circ}$ & 0.72 \\
$(\overline{112})$ oxidation, pre-RTA & $1.19 / 56^{\circ}$ & 0.57 & 0.68 \\
$(\overline{11} 2)$ oxidation, post-RTA & $1.23 / 46^{\circ}$ & 0.49 & 0.64 \\
$(\overline{11} 2)$ nitridation, pre-RTA & $1.15 / 56^{\circ}$ & 0.55 & 0.60 \\
$(\overline{11} 2)$ nitridation, post-RTA & $1.52 / 45^{\circ}$ & 0.62 & 0.76 \\
\hline \hline
\end{tabular}




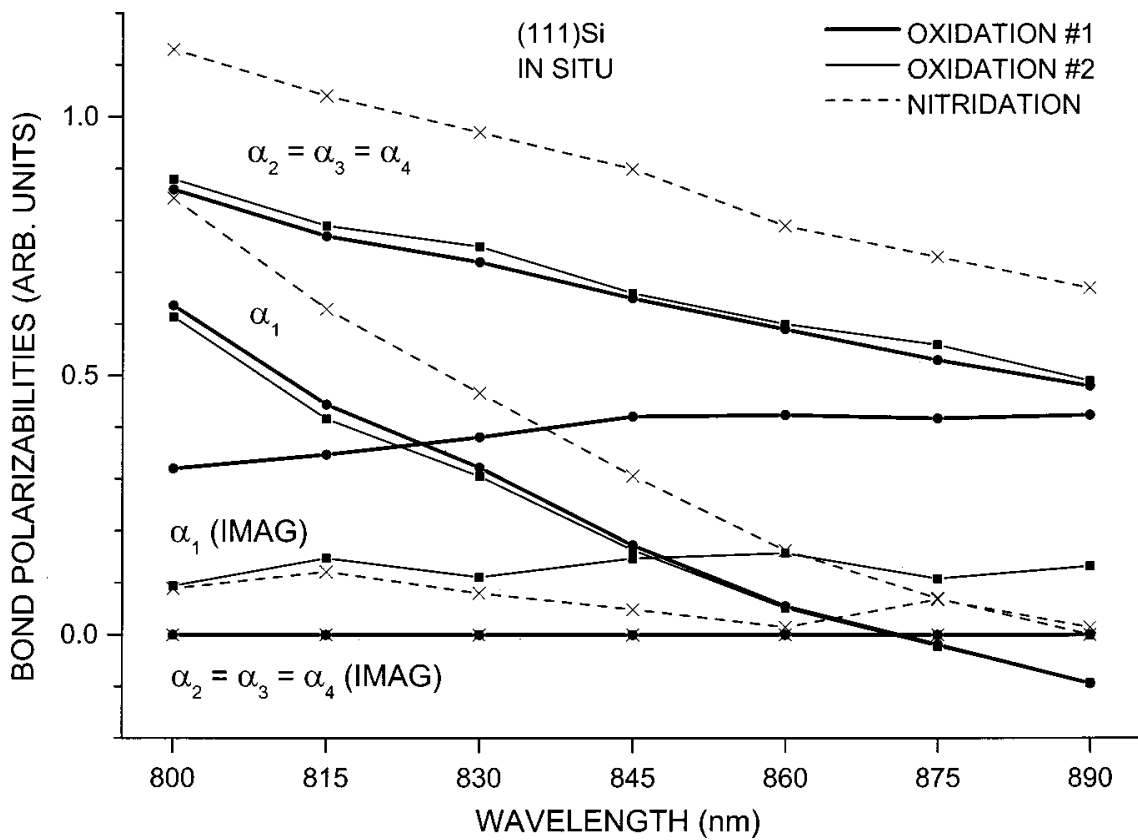

FIG. 5. Wavelength dependencies of the various hyperpolarizabilities obtained for singular (111) samples for two oxidations and a nitridation.
The situation is different for the sample cut $5^{\circ}$ off (111) toward (112). While the SHG absorption decreases with RTA for both oxidized and nitrided samples, the real part of the hyperpolarizability increases dramatically with RTA for the nitrided sample. The hyperpolarizability for this orientation is substantially less for the step bond, and the values move in opposite directions following RTA for the oxidized and nitrided interfaces, decreasing, and increasing, respectively. While we believe that RTA is increasing the perfection of the step in both cases, it is clear that the detailed bonding geometry must be taken into account before an increase is automatically considered to be the sign of improved bonding. As with the other orientation, the hyperpolarizabilities of the back bonds remain constant for the oxidized surface while those for the nitrided surface increase.

We conclude with an indication of the type of information that may be available when spectral SHG data become available. Figure 5 shows SHG anisotropies of the up and back bonds for three different singular (111) samples measured in situ after plasma processing. We chose this orientation because the three back bonds are equivalent, which means that only two classes of parameters need be considered. Data were obtained every $15 \mathrm{~nm}$ from 800 to $890 \mathrm{~nm}$ for two different oxidations and one nitridation run. The two oxidation runs are nominal duplicates of each other, allowing us to investigate reproducibility. It is clear from the heavy traces that the real parts of the hyperpolarizabilities $\alpha_{1}$ and $\alpha_{2}$ $=\alpha_{3}=\alpha_{4}$ of the two oxidation experiments are nearly equal. However, the SHG absorption for the up bond $\alpha_{1}$ shows a significant difference between the two oxidation runs, indicating that this feature is apparently rather sensitive to the details of specific plasma-processing runs.

Regarding nitridation, the real parts of all hyperpolarizabilities are consistently larger for the nitrided sample than for the oxidized samples. This is in contrast to the data given in Table I for the vicinal samples, but is an effect clearly outside experimental uncertainty. The absorption associated with the up bond appears to be generally less than that observed in oxidation.

The wavelength dependencies of the real parts of the nonzero contributions all follow essentially the same trend, decreasing monotonically with increasing wavelength. We consider first the back bonds, for which analysis is in principle easier. The decrease in the real part indicates the existence of absorption at wavelengths shorter than those accessible with our current apparatus. On the other hand, and given our phase reference, the imaginary part of the hyperpolarizability of the up bond appears to be approximately constant or even increasing slightly with decreasing wavelength as the real part crosses zero, indicating that its absorption may be reaching a maximum near or within the present spectral region. Given the limited wavelength range of these data these remarks are essentially speculation, but these data do indicate that considerable information should be available when SHG anisotropy measurements such as these are made and interpreted in the SBHM over an extended spectral range. Considering in addition that we can identify the hyperpolarizability of step bonds when dealing with vicinal surfaces, we should be able to form a rather complete picture of the absorption processes of these bonds at these interfaces. Similar investigation of dielectrics other than silicon oxides and nitrides should also be fruitful.

\section{CONCLUSION}

We have shown that a simplified bond-hyperpolarizability model (SBHM) provides a straightforward and mathematically complete and efficient way of representing SHG anisotropy data in terms of parameters that have direct physical meaning on the microscopic scale. The SBHM allows us to extract some of the previously inaccessible physics of SHG at interfaces, specifically providing direct evidence of SHG 
absorption and allowing us to determine the effective angles of incidence and observation. This in turn allows us to identify the side of the interface at which the observed SHG response originates. Connection between Fourier coefficients describing the different anisotropies and the properties of the bonds could now be made, if desired, thereby providing a more microscopic basis for previous work.

With the exception of Fig. 5, we have considered only data that have been obtained at single wavelengths. The data shown in Fig. 5 indicate that extension of this approach to SHG anisotropies measured over a wide wavelength range will provide spectral information that will be useful for more detailed analyses. This would be extremely interesting not only as a means of obtaining greater insight concerning the energy dependence and therefore the origin of the observed SHG absorption, but also for additionally critically assessing the SBHM model. In particular, an assessment of the effective angles of incidence and observation as a function of wavelength would provide additional insight into the specific interface region responsible for the SHG signal.

\section{ACKNOWLEDGMENTS}

It is a pleasure to acknowledge the Office of Naval Research for the original grant under which our SHG equipment was purchased and for continuing support. We would also like to acknowledge further support by the Army Research Office and the Max-Planck-Gesellschaft via an Award for International Cooperation.

${ }^{1}$ J. F. McGilp, Prog. Surf. Sci. 49, 1 (1995).

${ }^{2}$ G. Lüpke, Surf. Sci. Rep. 35, 75 (1999).
${ }^{3}$ Y. R. Shen, The Principles of Nonlinear Optics (Wiley, New York, 1984). ${ }^{4}$ W. Daum, H.-G. Krause, U. Reichel, and H. Ibach, Phys. Rev. Lett. 71, 1234 (1993).

${ }^{5}$ C. H. Bjorkman, C. E. Shearon, Jr., Y. Ma, T. Yasuda, G. Lucovsky, U. Emmerichs, C. Meyer, K. Leo, and H. Kurz, J. Vac. Sci. Technol. A 11, 964 (1993).

${ }^{6}$ C. H. Bjorkman, T. Yasuda, C. E. Shearon, Jr., Y. Ma, G. Lucovsky, U. Emmerichs, C. Meyer, K. Leo, and H. Kurz, J. Vac. Sci. Technol. B 11, 1521 (1993).

${ }^{7}$ G. Lüpke, D. J. Bottomley, and H. M. van Driel, Phys. Rev. B 47, 10389 (1993).

${ }^{8}$ G. Lüpke, D. J. Bottomley, and H. M. van Driel, J. Opt. Soc. Am. B 11, 33 (1994)

${ }^{9}$ J. I. Dadap, X. F. Hu, M. H. Anderson, M. C. Downer, J. K. Lowell, and O. A. Aksipetrov, Phys. Rev. B 53, R7607 (1996).

${ }^{10}$ C. Meyer, G. Lüpke, Z. G. Lũ, A. Götz, H. Kurz, and G. Lucovsky, J. Vac. Sci. Technol. B 14, 3107 (1996).

${ }^{11}$ S. T. Cundiff, W. H. Knox, F. H. Baumann, K. W. Evans-Lutterodt, and M. L. Green, J. Vac. Sci. Technol. A 16, 1730 (1998).

${ }^{12}$ G. Erley and W. Daum, Phys. Rev. B 58, R1734 (1998).

${ }^{13}$ J. E. Sipe, D. J. Moss, and H. M. van Driel, Phys. Rev. B 35, 1129 (1987).

${ }^{14}$ V. Mizrahi and J. E. Sipe, J. Opt. Soc. Am. B 5, 660 (1988).

${ }^{15}$ J. E. Sipe and A. I. Shkrebtii, Phys. Rev. B 61, 5337 (2000).

${ }^{16}$ See, e.g., M. Cini, Phys. Rev. B 43, 4792 (1991).

${ }^{17}$ P. P. Ewald, Dissertation, Munich, 1912; Ann. Phys. (Paris) 49, 1 (1916).

${ }^{18}$ C. W. Oseen, Ann. Phys. (Paris) 48, 1 (1915).

${ }^{19}$ G. D. Powell, J.-F. T. Wang, and D. E. Aspnes, Phys. Rev. B 65, 205320 (2002).

${ }^{20}$ N. Bloembergen and P. S. Pershan, Phys. Rev. 128, 606 (1962).

${ }^{21}$ N. Bloembergen, R. K. Chang, S. S. Jha, and C. H. Lee, Phys. Rev. 174, 813 (1968).

${ }^{22}$ B. F. Levine, Phys. Rev. B 7, 2600 (1973)

${ }^{23}$ W. L. Schaich and B. S. Mendoza, Phys. Rev. B 45, 14279 (1992).

${ }^{24}$ C. M. J. Wijers, P. L. de Boeij, C. W. van Hasselt, and Th. Rasing, Solid State Commun. 93, 17 (1995).

${ }^{25}$ C. H. Patterson, D. Weaire, and J. F. McGilp, J. Phys.: Condens. Matter 4, 4017 (1992).

${ }^{26}$ B. S. Mendoza and W. L. Mochán, Phys. Rev. 55, 2489 (1997).

${ }^{27}$ N. Arzate and B. S. Mendoza, Phys. Rev. B 63, 113303 (2001). 